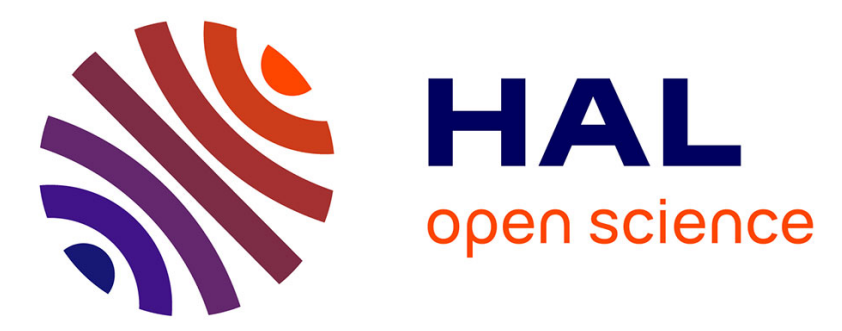

\title{
Spin-dependent transport in antiferromagnetic tunnel junctions
}

P. Merodio, A. Kalitsov, H. Béa, Vincent Baltz, M. Chshiev

\section{To cite this version:}

P. Merodio, A. Kalitsov, H. Béa, Vincent Baltz, M. Chshiev. Spin-dependent transport in antiferromagnetic tunnel junctions. Applied Physics Letters, 2014, 105, pp.122403. 10.1063/1.4896291. hal-01683647

\section{HAL Id: hal-01683647 https://hal.science/hal-01683647}

Submitted on 19 May 2019

HAL is a multi-disciplinary open access archive for the deposit and dissemination of scientific research documents, whether they are published or not. The documents may come from teaching and research institutions in France or abroad, or from public or private research centers.
L'archive ouverte pluridisciplinaire HAL, est destinée au dépôt et à la diffusion de documents scientifiques de niveau recherche, publiés ou non, émanant des établissements d'enseignement et de recherche français ou étrangers, des laboratoires publics ou privés. 


\title{
Spin-dependent transport in antiferromagnetic tunnel junctions
}

\author{
P. Merodio, ${ }^{1, a)}$ A. Kalitsov, ${ }^{1,2}$ H. Béa, ${ }^{1}$ V. Baltz, ${ }^{1}$ and M. Chshiev ${ }^{1, a)}$ \\ ${ }^{1}$ Univ. Grenoble Alpes, INAC-SPINTEC, F-38000 Grenoble, France; CNRS, SPINTEC, F-38000 Grenoble, \\ France; and CEA, INAC-SPINTEC, F-38000 Grenoble, France \\ ${ }^{2}$ MINT Center, University of Alabama, Box 870209, Tuscaloosa, Alabama 35487, USA
}

(Received 8 July 2014; accepted 8 September 2014; published online 22 September 2014)

\begin{abstract}
We investigate the behaviour of spin transfer torque (STT) and tunnelling magnetoresistance (TMR) in epitaxial antiferromagnetic-based tunnel junctions using tight binding calculations in the framework of the Keldysh formalism. We find that the STT out-of-plane component exhibits a staggered spatial distribution similar to its in-plane component. This behaviour is specific to the use of a tunnel barrier and significantly differs from the out-of-plane torques reported in previous works using a metallic spacer. Additionally, we show that unlike conventional ferromagnetic-based tunnel junctions, the TMR can increase with applied bias and reach values comparable to typical magnetoresistances found for usual spin valves. (C) 2014 AIP Publishing LLC.
\end{abstract}

[http://dx.doi.org/10.1063/1.4896291]

Antiferromagnets (AFs)-based spintronics is a branch of science that explores spin dependent transport devices using AFs instead of ferromagnets $(F){ }^{1,2}$ It is currently considered as a significant exploratory topic in spintronics ${ }^{3-6}$ since AFs exhibit no stray fields, which is beneficial for ultimate downsize scalability. In particular, a first theoretical toy model showed AF spin transfer torque (STT) and giant magnetoresistance (GMR) for metallic AF/PM/AF multilayers, ${ }^{7}$ where PM stands for a paramagnetic metallic spacer. The authors considered crystalline uncompensated $\mathrm{F}$ monolayers with staggered AF order (i.e., two alternating $\mathrm{F}$ sublattices with opposite magnetizations). Furthermore, unlike the pioneering theoretical works on STT in F multilayers, ${ }^{8,9}$ which predict torques exerted by a spin polarized current close to the interface between a F and a nonmagnetic metal, STT is expected to act cooperatively through the entire volume of the AF electrodes. This feature together with the absence of shape anisotropy in AFs explain that lower critical currents for magnetization switching are predicted for epitaxial AFs compared to the typical values for Fs.

The STT picture proposed in Ref. 7 arises from changes in the exchange field experienced by localized moments due to their magnetic interaction with nonequilibrium spin densities originated by conduction electrons. This is in contrast with the widely used theoretical framework for $\mathrm{F}$ systems, where the basic idea is that the precession of an electron about the magnetization of a $\mathrm{F}$ yields to a change in this magnetization by conservation of angular momentum, equal to the imbalance of inward and outward spin fluxes. Due to the vanishing magnetization in AF metals, this formalism can only be applied locally.

STT has been experimentally shown, for instance, with layered ferromagnetic contacts with metallic spacers ${ }^{10,11}$ and with a scanning tunneling microscope. ${ }^{12}$ These experimental works are based on the variation in the resistance of the different $\mathrm{F}$ structures. However, the difficulty to pin the order parameter of an $\mathrm{AF}$ element along a reference direction together with the dwarfed magnetoresistance observed in $\mathrm{AF} /$

a)Electronic addresses: mair.chshiev@cea.fr and pablo.merodiocamara@cea.fr
$\mathrm{PM} / \mathrm{AF}$ multilayers ${ }^{13}$ makes the observation of STT in AF a technological challenge. Indirect mechanisms such as the study of exchange bias variations at F/AF interfaces due to the effect of a spin polarized current on the spin orientation of the AF interfacial layer ${ }^{14}$ are thus a convenient tool for studying STT in AF.

Apart from spin-valve structures using metallic spacers, usual F-based spintronics takes advantage since long of tunnel barriers (B). ${ }^{15}$ Whereas GMR relies on spin dependent scattering at interfaces, tunnel magnetoresistance (TMR) rather relates to spin dependent densities of states. In addition, tunnel barriers filter the wave vectors' angles of incidence of incoming electrons, which lowers the effect of dephasing in three dimensional systems (3-D). ${ }^{16}$ In effect, the tunneling current distribution over the two-dimensional Brillouin zone (corresponding to the wave vector component parallel to the barrier interface, $\mathbf{k}_{\|}$) is strongly localized in certain regions. For instance, STT is mostly determined by electrons with perpendicular incidence and decreases quickly with $k_{\|}$in $\mathrm{F}$ tunnel junctions. ${ }^{17}$

We investigate the behaviour of STT and TMR in AFbased tunnel junctions using tight binding calculations in the framework of the Keldysh formalism both for 1-D and 3-D geometries. Due to the use of tunnel barriers, we anticipate unusual behaviours compared to AF-based spin-valves with metallic spacers, as described below.

Fig. 1 sketches a typical tunnel junction layer structure. The semi-infinite leads are modelled as a chain of uncompensated localized spins with translational invariance in the plane perpendicular to the electron flow (y axis). We use a single band tight binding Hamiltonian for the itinerant electrons with a hopping parameter $t=-1 \mathrm{eV}$ in all regions. The insulating spacer is a barrier of $\mathrm{N}_{\mathrm{B}}$ sites (respectively layers) for 1-D (respectively 3-D) with a spin-independent on-site energy $\varepsilon_{\mathrm{B}}=5 \mathrm{eV}$. The spin-dependent on-site energy in the leads is the sum of the $s$-like orbital energy $\varepsilon_{0}$ and the spin splitting $\Delta$, which accounts for the $s$ - $d$ magnetic interaction between itinerant spins and localized moments. The absolute value of the spin splitting $\Delta$ is constant, but alternates in sign for a given spin from one site(layer) to the next one due to the alternating orientation of the localized magnetic 

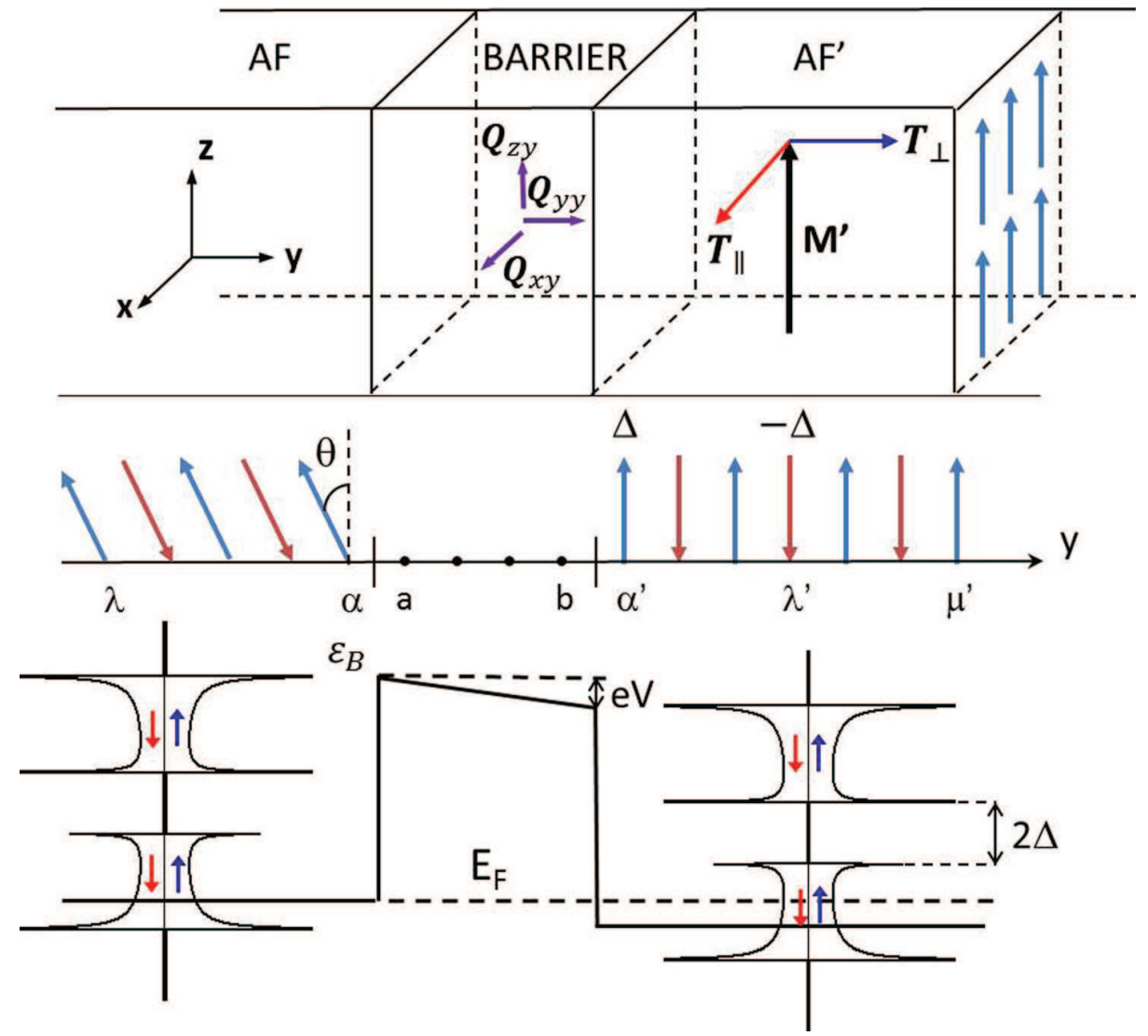

FIG. 1. Layouts of the tunnel junction modified from Ref. 8 for AF leads. (Top) Scheme of the left and right semi-infinite AF electrodes separated by the nonmagnetic insulating spacer of $\mathrm{N}_{\mathrm{B}}$ atomic layers. The order parameter $\mathrm{M}^{\prime}$ of the right lead points along the $z$ direction, while the order parameter $\mathrm{M}$ of the left lead is rotated by an angle $\theta$ around the y axis in the plane parallel to the AF/B interface. The on-site torques delivered in the right lead are represented by the in-plane $\left(T_{\|}\right)$and out-of-plane $\left(T_{\perp}\right)$ components. The vector decomposition of the spin current densities is depicted in the barrier $\left(\mathrm{Q}_{\mathrm{ij}}\right)$. (Middle) Schematic illustration of the two AF sublattices of up and down localized spins whose magnetic interaction with itinerant electrons is modeled by the staggered spin splitting $\Delta$. Sites in the left lead, barrier, and right lead are denoted, respectively, by unprimed Greek, Latin, and primed Greek letters. (Bottom) Schematic picture of the density of states in the AF leads and the potential profile within the junction. The lower and upper bands for a given spin are separated by a gap of $2 \Delta$. $\varepsilon_{B}$ is the spin-independent on-site energy in the barrier, and $\mathrm{V}$ is the potential applied through the junction. The Fermi level is set at $\mathrm{E}_{\mathrm{F}}=0$ and is indicated by the lower dashed line.

moments within the AF leads. In particular, localized up (similarly for down) spins build up a magnetic sublattice in which the spin-dependent on-site energy reads $\varepsilon^{\uparrow(\downarrow)}=\varepsilon_{0}-(+) \Delta$. Thus, the magnetic properties of each AF are embedded in the spin-splitting $\Delta$. In addition, the existence of two sublattices within the AF leads opens a gap in the density of states (DOS) equal to $2 \Delta$ (see Fig. 1). However, since AFs do not exhibit any macroscopic magnetization, majority and minority bands are not split.

The charge current density $J$ is calculated in the right lead utilizing the density operator or non-equilibrium lesser Green function $\hat{G}^{<}$, which is computed here in the framework of the Keldysh formalism considering ballistic transport ${ }^{18}$

$$
I=\frac{e t}{8 \pi^{3} \hbar} \int \operatorname{Tr}_{\sigma}\left[\hat{G}_{\lambda^{\prime}+1, \lambda^{\prime}}^{<\sigma, \sigma^{\prime}}-\hat{G}_{\lambda^{\prime}, \lambda^{\prime}+1}^{<\sigma, \sigma^{\prime}}\right] d E d \mathbf{k}_{\|},
$$

where $e$ is the elementary charge and $\mathbf{k}_{\|}$labels the Bloch state in each translationally invariant layer. Since coherent transport is considered here, we assume transverse momentum conservation. In the case of a 1-D junction, the prefactor $\frac{e t}{8 \pi^{3} \hbar}$ in Eq. (1) becomes $\frac{e t}{2 \pi \hbar}$ since the integration in the $\mathbf{k}_{\|}$ states is not necessary.

The essential block to calculate the projections of the lesser Green function $\hat{G}_{p, q}^{<}$into layers $p$ and $q$ for the whole structure is the retarded Green function $\hat{g}_{p, q}^{r}$ for isolated leads and barrier. In particular, the retarded Green function for the isolated semi-infinite AF leads is obtained analytically by renormalized perturbation expansion, considering the 1-D AF chain as a Bethe lattice with connectivity $K=1 .{ }^{19}$

The local torque is computed from the discrete divergence of the spin current density $\hat{Q}$

$$
\boldsymbol{T}_{\lambda^{\prime}}=-\nabla \cdot \hat{Q}=\hat{Q}_{\lambda^{\prime}-1, \lambda^{\prime}}-\hat{Q}_{\lambda^{\prime}, \lambda^{\prime}+1}=\frac{t}{16 \pi^{3}} \int \operatorname{Tr}_{\sigma}\left[\left(\hat{G}_{\lambda^{\prime}, \lambda^{\prime}-1}^{<\sigma, \sigma^{\prime}}-\hat{G}_{\lambda^{\prime}-1, \lambda^{\prime}}^{<\sigma, \sigma^{\prime}}-\hat{G}_{\lambda^{\prime}+1, \lambda^{\prime}}^{<\sigma, \sigma^{\prime}}+\hat{G}_{\lambda^{\prime}, \lambda^{\prime}+1}^{<\sigma, \sigma^{\prime}}\right) \cdot \boldsymbol{\sigma}\right] d E d \mathbf{k}_{\|} \cdot
$$




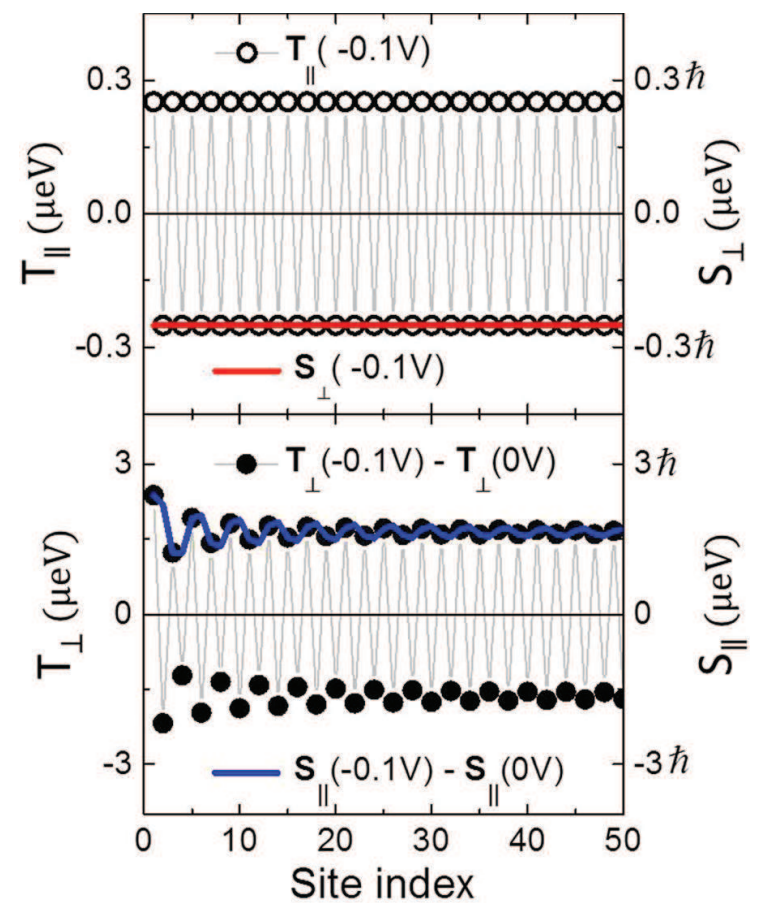

FIG. 2. Computed spatial distribution of the in-plane and out-of-plane components of the voltage-induced torque $\left(\mathrm{T}_{\|}\right.$and $\left.\mathrm{T}_{\perp}\right)$ and spin density $\left(S_{\perp}\right.$ and $\left.S_{\|}\right)$for a $1-\mathrm{D} \mathrm{F} / \mathrm{B} / \mathrm{AF}$ junction with $\theta=\pi / 2, \mathrm{~V}=-0.1 \mathrm{~V}, \varepsilon_{0}=1.5 \mathrm{eV}$, and $\Delta=0.5 \mathrm{eV}$ for both $\mathrm{F}$ and $\mathrm{AF}$ leads. The local out-of-plane torque $\mathrm{T}_{\perp}(0 \mathrm{~V})$ and in-plane spin density $S_{\|}(0 \mathrm{~V})$ at zero bias were subtracted to obtain the out-of-plane torque and the in-plane spin accumulation, respectively (see Fig. 3 for more details). Note that the equilibrium in-plane torque $T_{\|}(0 \mathrm{~V})$ and related out-of-plane spin density $S_{\perp}(0 \mathrm{~V})$ are zero. The red and blue thick lines representing the out-of-plane (top) and in-plane (bottom) spin densities, respectively, refer to the right-hand ordinate.

In addition, the equivalence of torques obtained from the spin current and from the exchange field in the $z$ direction is addressed by the computation of the local spin accumulation $\mathbf{S}_{\lambda^{\prime}}$

$$
\mathbf{S}_{\lambda^{\prime}}=\frac{-i \hbar}{16 \pi^{3}} \int \operatorname{Tr}_{\sigma}\left[\hat{G}_{\lambda^{\prime}, \lambda^{\prime}}^{<\sigma, \sigma^{\prime}} \cdot \boldsymbol{\sigma}\right] d E d \mathbf{k}_{\|}
$$

where $\boldsymbol{\sigma}$ is the vector of Pauli matrices. The prefactors in Eqs. (2) and (3) are replaced by $\frac{t}{4 \pi}$ and $\frac{-i \hbar}{4 \pi}$, respectively, for 1-D junctions, where the integral is performed in the energy domain only.

The in-plane and out-of-plane torque components defined in Fig. 1 are thus originated by the out-of-plane and in-plane components of spin accumulation, respectively,

$$
\begin{gathered}
T_{\lambda^{\prime}}^{\|}=(-1)^{\lambda^{\prime}} \times \frac{2 \Delta_{\lambda^{\prime}}}{\hbar} \times S_{\lambda^{\prime}}^{\perp}, \\
T_{\lambda^{\prime}}^{\perp}=(-1)^{\lambda^{\prime}+1} \times \frac{2 \Delta_{\lambda^{\prime}}}{\hbar} \times S_{\lambda^{\prime}}^{\|} .
\end{gathered}
$$

In Fig. 2, we show the voltage-induced part of in-plane (open circles) and out-of-plane (solid circles) spin torque spatial distributions within the right lead of a 1-D F/B/AF junction. The in-plane component exhibits the perfectly staggered distribution that was previously observed by Núñez et l. $^{7}$ for 3-D AF-based spin valves. The alternating sign of the torque is produced by the alternating localized magnetic moments of each sublattice in the AF lead. These alternating moments are responsible for the alternating exchange field seen by transport electrons. In turn, as described in Eq. (4), the constant out-of-plane spin density originated by these transport electrons couple to the alternating exchange field, which leads to the alternating sign of the torque from one site to the adjacent site.

However, unlike the random distribution of the out-ofplane torque shown with such metallic spacer, in the case of an insulating spacer analysed here, the non-equilibrium part of this torque component is also staggered. This is valid regardless of the left lead's magnetic order since we observed similar behaviour using AF and FI leads as polarisers. Furthermore, the staggered spatial distribution of the out-of-plane torque survives the $\mathbf{k}_{||}$integration for 3-D geometries. Together with the reflection-based polarizing mechanisms previously reported for $\mathrm{AF}^{6,7}$ we emphasize that despite the overall DOS in AF remains unpolarized at the Fermi level, the local interfacial DOS becomes spin polarized giving rise to TMR and STT. Thus, STT and TMR behavior is strongly influenced by the two uncompensated layers next to the insulating barrier.

The red and blue thick lines in Fig. 2 (top and bottom panels, respectively) represent the out-of-plane and in-plane spin accumulations in each site of the right lead. By taking into account the scale factors given by the on-site spin-splitting and the sign of the $s$ - $d$ local magnetic interaction (due to the staggered orientation of each localized moment), the results represented in Fig. 2 demonstrate that spin accumulation drives the torques. A constant out-of-plane spin accumulation leads to a staggered in-plane torque. In turn, the in-plane spin accumulation oscillates slightly around a constant value, so that the out-of-plane torque exerted in each site of sublattice A oscillates converging to $1.62 \mu \mathrm{eV}$ (the limiting torque in the bulk) and the torque deposited in sublattice B oscillates around the same value with opposite sign. We ascribe the different spatial distributions of out-ofplane torque and in-plane spin density in the right lead for spin valves and tunnel junctions to the multiple spindependent reflections that take place in the metallic spacer but not in the insulating barrier. In effect, in the case of tunnel junctions, the evanescent waves decaying exponentially do not stay in the barrier, which reduces considerably the quantum interference between spin-dependent leftward and rightward electrons' wave functions. Thus, in the particular case of the in plane spin component, quantum interference in a metallic spacer may lead to a non-coherent spatial distribution of the in-plane spin density (and consequently out-of-plane torque) both in the spacer and in the right lead. In contrast, as analogous staggered spatial distributions of the in-plane torque are present in both systems, the $\mathbf{k}_{\|}$-filtering effect of tunnel junctions is unlikely to be at the origin of the different out-of-plane torque behaviour, since the spin precession dephasing of the different $\mathbf{k}_{\|}$states would affect similarly to both torque components.

In order to illustrate the effect of the equilibrium contribution to the out-of-plane torque, we plot in Fig. 3 the spatial distribution of the out-of-plane torque at zero bias (bottom), and the total out-of-plane torque at $-0.1 \mathrm{~V}$ (top) in a 1-D F/ $\mathrm{B} / \mathrm{AF}$ junction. The subtraction of the equilibrium torque to 


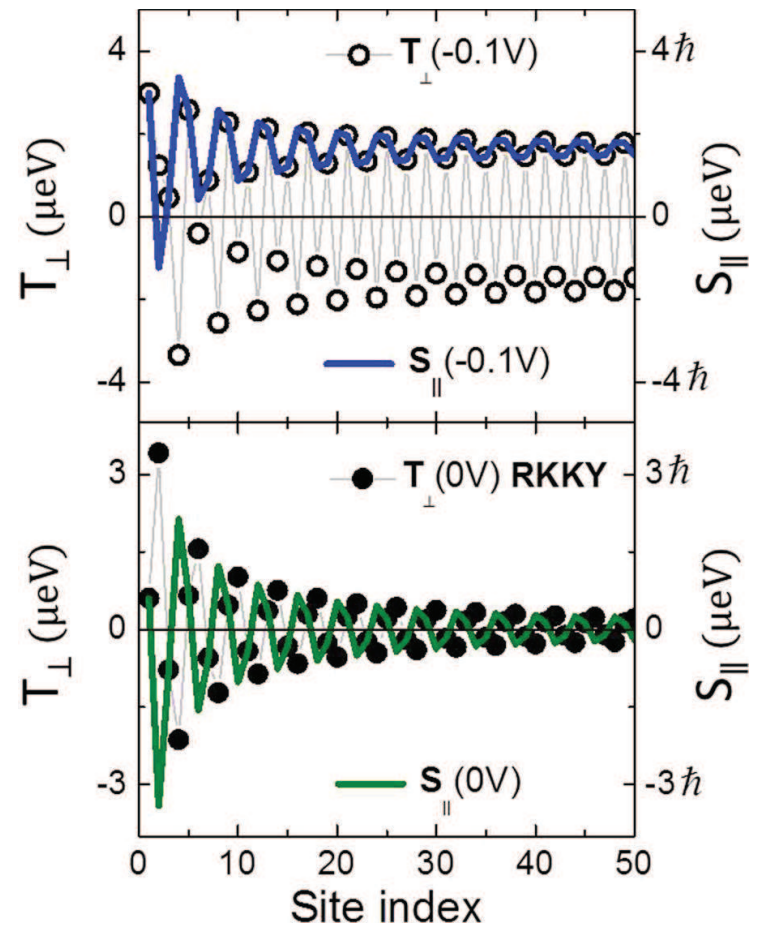

FIG. 3. Computed spatial distribution of the total out-of-plane torque $T_{\perp}$ and the total in-plane spin density $S_{\|}$for a $1-\mathrm{D} \mathrm{F} / \mathrm{B} / \mathrm{AF}$ junction with $\theta=\pi / 2$ at $-0.1 \mathrm{~V}$ and $0 \mathrm{~V}$ (RKKY interaction) with $\varepsilon_{0}=1.5 \mathrm{eV}$ and $\Delta=0.5 \mathrm{eV}$ for both $\mathrm{F}$ and $\mathrm{AF}$ leads. The blue and green thick lines representing, respectively, the in-plane spin densities at $-0.1 \mathrm{~V}$ (top) and $0 \mathrm{~V}$ (bottom) refer to the right-hand ordinate.

the total torque of Fig. 3 gave the non-equilibrium torque represented in Fig. 2. The former accounts for the Ruderman-Kittel-Kasuya-Yosida (RKKY) interaction and is not associated with charge transport. This RKKY conduction-electron-mediated interaction, often referred to as interlayer exchange coupling (IEC) between localized spins in the left and right leads, ${ }^{20}$ is comparable in magnitude to the transport-induced out-of-plane torque near the $\mathrm{B} / \mathrm{AF}$ interface (even higher at certain points). It decays so gradually that the total out-of-plane torque is strongly affected deep into the electrode: subtracting the IEC to the total out-of-plane torque smoothes out its own oscillations (which are highlighted by the blue curve representing the inplane spin accumulation in the upper plot of Fig. 3), leading to the flatter staggered torque shown in the lower plot of Fig. 2. Unlike the voltage-induced part of the out-of-plane torque, the torque exerted at zero bias in each individual sublattice of the right AF lead as well as the in-plane spin accumulation are staggered. Consequently, the equilibrium out-of-plane torque spatial distribution is not staggered since the sign of the local torque and spin density alternates every two sites (owning to each of the sublattices). In contrast, the voltage-induced contribution in 3-D AF-based tunnel junction (not shown) is not significantly affected by the IEC beyond the first layers next to the B/AF interface. Although the profile of the total out-of-plane torque is abruptly distorted near this interface due to a very high value of the IEC, this equilibrium contribution is radically damped. Interestingly, we found again a similar STT behaviour regardless of the magnetic nature of the polariser.
We next examine the voltage dependence of the charge current density for different angles $\theta$ of the left lead order parameter (see Fig. 1 for the definition of $\theta$ ) and TMR in a 3-D AF-based tunnel junction. The TMR and its voltage dependence behavior are, in general, defined by the electronic structure of the leads around the Fermi level. Namely, the interfacial DOS determines the spin polarization and the conductance. The energy dependence of interfacial DOS results in the variation of the tunnel current and TMR with the applied voltage. $^{21}$

Fig. 4(b) shows the charge current density as a function of applied bias for antiparallel, perpendicular, and parallel magnetic states of left and right AF leads (the magnetic states are defined here as the relative orientation of the two layers of uncompensated spins at the $\mathrm{AF} / \mathrm{B}$ and $\mathrm{B} / \mathrm{AF}$ interfaces). The same features are shown in Fig. 4(a) for a conventional F-based tunnel junction. In the low bias region, where the charge density current can be considered as proportional to the applied bias, ${ }^{22}$ differences between the three magnetic states are sensible only in the F case. In contrast, for the AF case, the three configurations can only be distinguished for voltages higher than a threshold of around $0.4 \mathrm{~V}$. Higher voltages produce charge current saturation and decrease at very different rates for the three magnetic configurations. This is clearly illustrated by the TMR voltage dependence depicted in Fig. 4(d). The position of the threshold might be ascribed to the curvature change of the AF interfacial LDOS at the Fermi level when a voltage is applied, which amounts to a steep change in the number of states available for one of the spin channels. Further, investigations are however necessary in order to clarify this point. For bias below $0.4 \mathrm{~V}$, the TMR is negligible (negative TMR is also found, for instance, in the case of FI leads due to negative spin polarization ${ }^{23}$ ), but interestingly it presents a dramatic increase with voltage from the threshold, reaching values as high as $90 \%$ for a bias of $0.9 \mathrm{~V}$. This bias dependence of the TMR in AF-based tunnel junctions is opposite to the usual case of ordinary F-based tunnel junctions, where TMR reaches very high values, but decreases significantly with applied voltage as shown in Fig. 4(c). Different trends were found for different AF. For instance, a decreasing TMR with voltage was observed for an $\mathrm{AF}$ with $\varepsilon_{0}=1.5 \mathrm{eV}$ and $\Delta=0.5 \mathrm{eV}$. The effect of this change in the electronic and magnetic parameters on the lower band of the AF lead amounts to a slight down shift of the lower band edge. This demonstrates the extreme sensitivity of the current properties in AF-based tunnel junctions to the AF electronic band. We found however a monotonic dependence of TMR with applied voltage, in contrast with the more complex oscillatory behavior of tunneling anisotropic magnetoresistance observed in single Co atoms adsorbed on double Fe films, ${ }^{24}$ explained in terms of the LDOS of the metallic system.

In summary, the spatial distribution of the STT in epitaxial AF-based tunnel junctions was analysed in terms of local spin accumulation and the discrete divergence of the spin current transverse component. The resulting voltageinduced part of the out-of plane torque (as well as the total out-of plane torque in the bulk) deposited in an AF lead is found to be perfectly staggered, in contrast with the random behaviour previously reported for a metallic spacer. The 


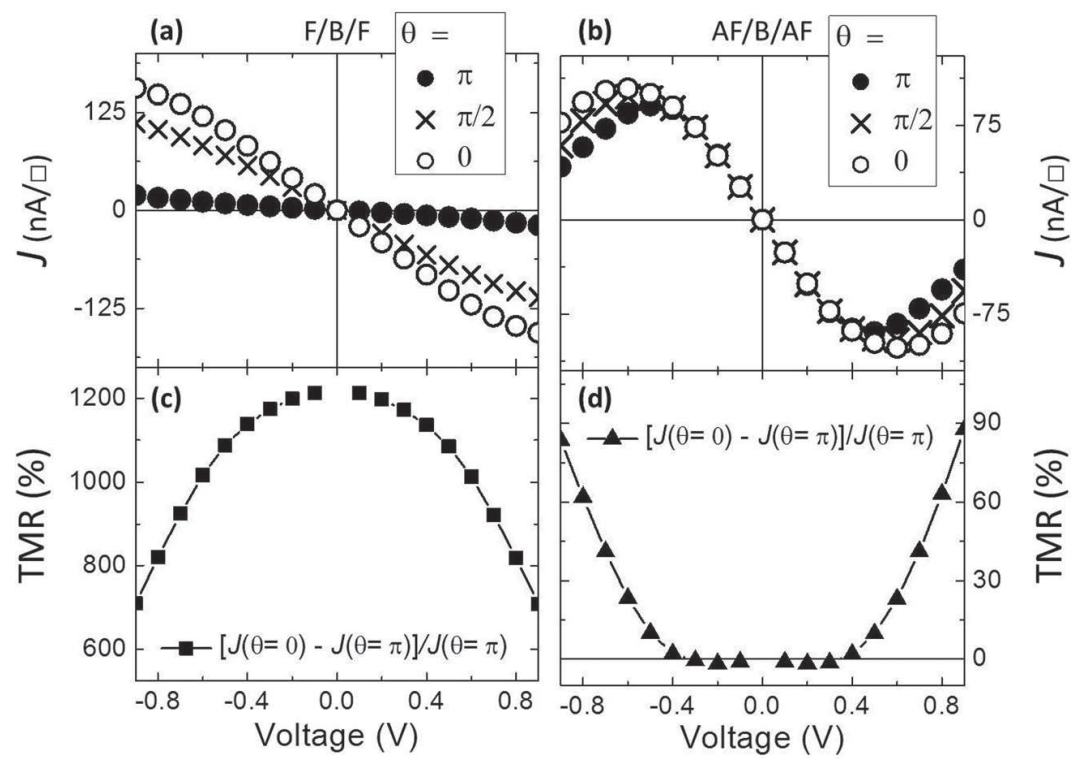

FIG. 4. Calculated voltage dependence of the charge current density I in nA by unit of surface $\square$ (top) and TMR (bottom) for three different angles $\theta$ in (a) and (c) a 3-D F/B/F tunnel junction with $\varepsilon_{0}=1.75 \mathrm{eV}, \Delta=0.75 \mathrm{eV}$ in both $\mathrm{F}$ leads, and in (b) and (d) a 3-D AF/B/ AF tunnel junction with $\varepsilon_{0}=2 \mathrm{eV}$ and $\Delta=1 \mathrm{eV}$ in both AF leads.

STT spatial pattern is independent of the magnetic nature of the left lead, which points out that the main role of polariser is played by closest spins next to the barrier's left interface. In addition, we showed that unlike conventional F-based tunnel junctions, AF-based tunnel junctions can show monotonically increasing TMR with voltage, reaching values as high as $90 \%$ for a bias of $0.9 \mathrm{~V}$.

${ }^{1}$ J. Basset, A. Sharma, Z. Wei, J. Bass, and M. Tsoi, Proc. SPIE 7036, 703605 (2008).

${ }^{2}$ A. H. MacDonald and M. Tsoi, Philos. Trans. R. Soc. 369, 3098 (2011).

${ }^{3}$ R. A. Duine, Nat. Mater. 10, 344 (2011).

${ }^{4}$ J. Sinova and I. Žutić, Nat. Mater. 11, 368 (2012).

${ }^{5}$ A. Brataas, A. D. Kent, and H. Ohno, Nat. Mater. 11, 372 (2012).

${ }^{6}$ H. B. M. Saidaoui, A. Manchon, and X. Waintal, Phys. Rev. B 89, 174430 (2014).

${ }^{7}$ A. S. Núñez, R. A. Duine, P. Haney, and A. H. MacDonald, Phys. Rev. B 73, $214426(2006)$

${ }^{8}$ J. C. Slonczewski, J. Magn. Mater. 159, L1 (1996).

${ }^{9}$ L. Berger, Phys. Rev. B 54, 9353 (1996).

${ }^{10}$ M. Tsoi, A. G. M. Jansen, J. Bass, W.-C. Chiang, M. Seck, V. Tsoi, and P. Wyder, Phys. Rev. Lett. 80, 4281 (1998).

${ }^{11}$ E. B. Myers, D. C. Ralph, J. A. Katine, R. N. Louie, and R. A. Buhrman, Science 285, 867 (1999).
${ }^{12}$ S. Krause, L. Berbil-Bautista, G. Herzog, M. Bode, and R. Wiesendanger, Science 317, 1537 (2007).

${ }^{13}$ L. Wang, S. G. Wang, S. Rizwan, Q. H. Qin, and X. F. Han, Appl. Phys. Lett. 95, 152512 (2009).

${ }^{14}$ Z. Wei, A. Sharma, A. S. Núñez, P. M. Haney, R. A. Duine, J. Bass, A. H. MacDonald, and M. Tsoi, Phys. Rev. Lett. 98, 116603 (2007).

${ }^{15}$ J. S. Moodera, L. R. Kinder, T. M. Wong, and R. Meserveyet, Phys. Rev. Lett. 74, 3273 (1995).

${ }^{16}$ D. C. Ralph and M. D. Stiles, J. Magn. Magn. Mater. 320, 1190-1216 (2008).

${ }^{17}$ A. Manchon, N. Ryzhanova, A. Vedyayev, M. Chschiev, and B. Dieny, J. Phys.: Condens. Matter 20, 145208 (2008).

${ }^{18}$ A. Kalitsov, M. Chshiev, I. Theodonis, N. Kioussis, and W. H. Butler, Phys. Rev. B 79, 174416 (2009).

${ }^{19}$ E. N. Economou, Green's Functions in Quantum Physics, Springer Series in Solid-State Sciences Vol. 7 (Springer, 2006).

${ }^{20}$ M. A. Ruderman and C. Kittel, Phys. Rev. 96, 99-102 (1954); T. Kasuya, Prog. Theory Phys. 16, 45-57 (1956); K. Yosida, Phys. Rev. 106, 893-898 (1957).

${ }^{21}$ E. Y. Tsymbal, O. N. Mryasov, and P. R. LeClair, J. Phys.: Condens. Matter 15, R109 (2003).

${ }^{22} \mathrm{H}$. Bruus and K. Flensberg, Many-Body Quantum Theory in Condensed Matter Physics: An Introduction (Oxford Graduate Texts, 2004).

${ }^{23}$ C. Kaiser and S. S. P. Parkin, Appl. Phys. Lett. 88, 112511 (2006).

${ }^{24}$ N. Néel, S. Schröder, N. Ruppelt, P. Ferriani, J. Kröger, R. Berndt, and S. Heinze, Phys. Rev. Lett. 110, 037202 (2013). 